\title{
Pore Channel Orientation in Self-Assembled Inorganic Mesostructures
}

\author{
D. A. Blom, L. F. Allard, M. Z. Hu, V. F. de Almeida, S. Dai, C. Tsouris, and Z. Zhang \\ Oak Ridge National Laboratory, PO Box 2008, Oak Ridge, TN 37831
}

Bottom-up fabrication of micro- or nano-systems demands controlled buildup of inorganic precursor materials in the desired location, shape, and quantity. One fundamental and technologically significant structure consists of an inorganic film layer on a solid substrate. Ideally, the layer contains highly ordered and oriented arrays of nano- or meso-pore channels perpendicular to the substrate surface. If this model nanostructure can be fabricated in large area with controllable thickness, it will have a great impact upon many applications such as photovoltaic and photoluminescent devices, nanoelectronics, fuel cells, nonlinear optical devices, membranes for selective gas separation and catalysis, sensors, etc. Active research at Oak Ridge National Laboratory aims at developing a capability in controlled formation of such inorganic nanostructures using "soft" molecular self-assembly. [1,2,3] However, self-assembly per se is not sufficient; to date, it has only achieved short-range ordered mesostructures lacking control of orientation. There is a need to develop the engineering means to manipulate self-assembly processes and achieve long-range order and orientation in templated inorganic nanomaterials.

High-resolution electron microscopy is effectively employed in concert with synthesis efforts to study the formation of titania and silica mesostructures formed by templating with block copolymers. A Hitachi HF-2000 FE-TEM and Hitachi HD-2000 dedicated STEM have been used as screening tools in identifying conditions that could induce the self-assembly of desired mesostructures. Through an iterative process of experimentation and microscopic analysis, we have determined process parameters that significantly affect the formation of self-assembled, highly ordered mesostructures. An example of the various mesostructures that have been synthesized is shown in Figure 1. This figure presents a titania mesostructure containing parallel straight or curved patterns with lattice spacing of $\sim 10 \mathrm{~nm}$. The dark lines in Figure 1 indicate titania nanophases, which contain 2-3 nm nanocrystals and amorphous titania that nucleate and grow in the hydrophilic microphase of the block copolymer.

The ordered inorganic channels shown in Figure 1 mostly lie down along the substrate surface. A fundamental challenge is to make them stand up. We are exploring means to induce "directed" molecular assembly. In some experimental conditions, hexagonal phases (Figure 2, viewed along arrays of parallel pore channels) seem to appear, indicating possible orientation of pore channels perpendicular to the substrate surface or to the film layer. Lines connecting the neighboring black spots in Figure 2 are drawn to assist the visualization. It is worth noting that the hexagonal shapes are not perfect because of distortion or possible damage of the mesoporous film layer under electron beam irradiation. At this point, the relative importance of various process parameters on the selfassembly phenomena is not clear. Future studies employing high-resolution microscopy, in conjunction with other experimental tools, will aim to clarify the controlling factors in mesostructure orientation and to develop strategies to expand the ability to synthesize desired mesostructures. [4] 


\section{References}

[1] Daniel M. Dabbs and Ilhan A. Aksay, Annu. Rev. Phys. Chem., 51, 60 (2000).

[2] Gisle Oye, Johan Sjoblom, and Michael Stocker, Adv. Colloid Inter. Sci., 89-90, 439 (2001).

[3] Jackie Y. Ying, AIChE J., 46, 1902 (2000).

[4] Research sponsored by the Divisions of Chemical Science and Materials Science of the Office of Basic Energy Science, U.S. Department of Energy and by the Asst. Secretary for Energy Efficiency and Renewable Energy, Office of Freedom CAR and Vehicle Technologies, as part of the High Temperature Materials Laboratory User Program, Oak Ridge National Laboratory, managed by UTBattelle, LLC, for the U.S. Department of Energy.

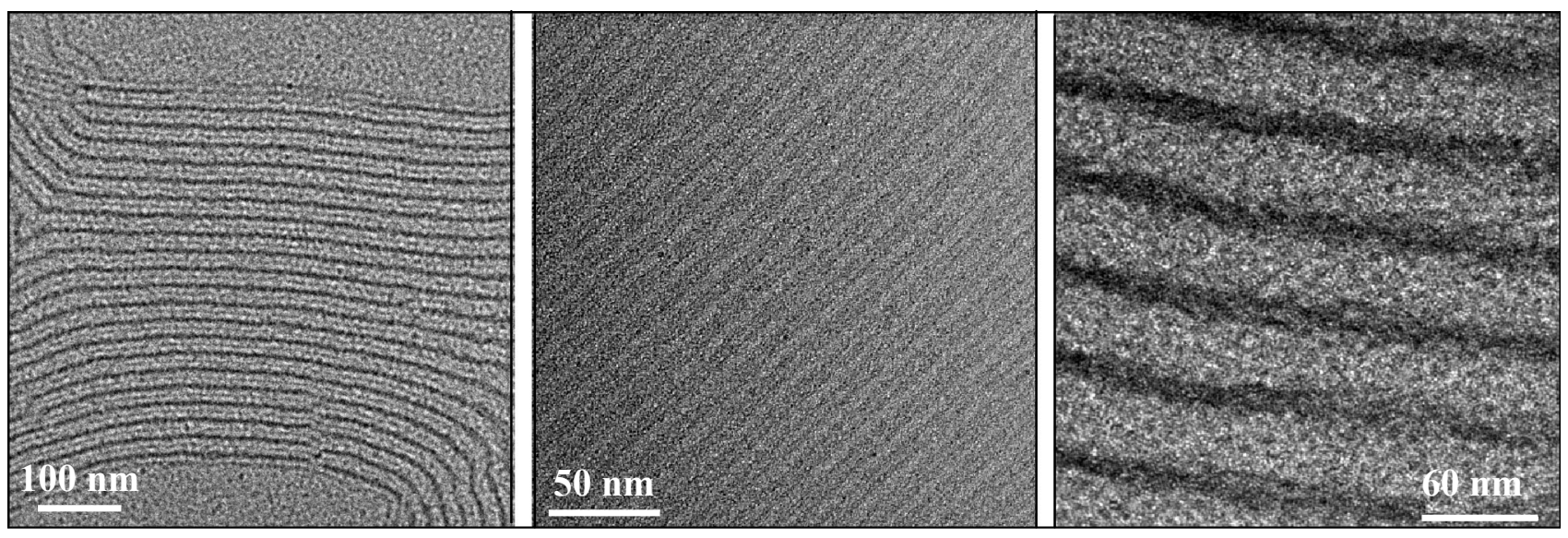

FIG. 1. High-resolution TEM images of titania layer mesostructures containing parallel pore channels with spacing of $\sim 10 \mathrm{~nm}$. Dark pore channel lines correspond to titania phase.

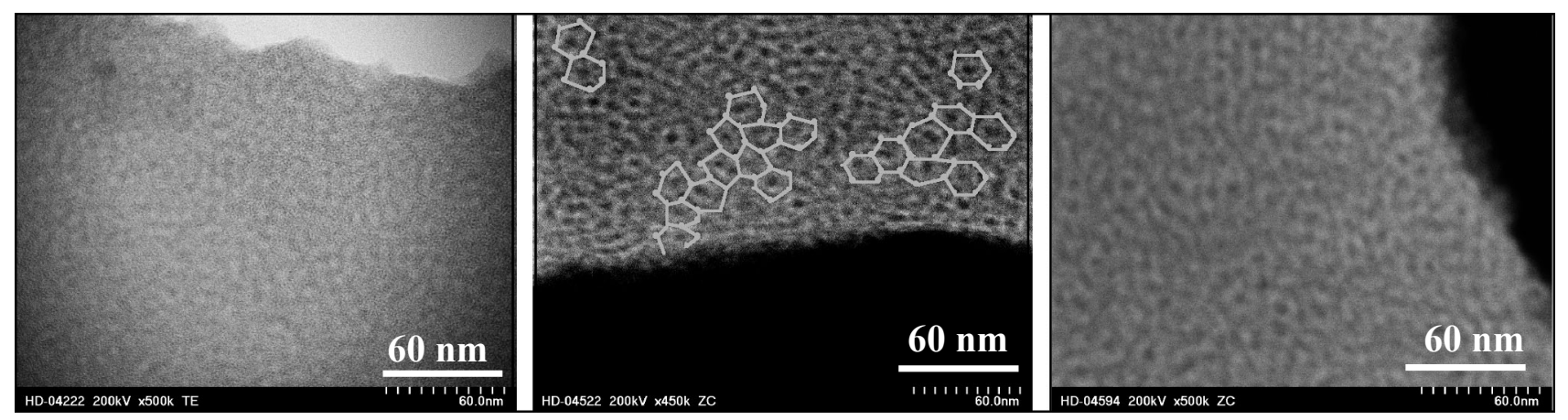

FIG. 2. STEM images of mesostructured titania showing possible hexagonal phases (TEtransmission mode; $Z C-Z$-contrast mode, brighter areas correspond to titania phase). 